\title{
Design Consideration for Developing Bio-potential Instrumentation Amplifier Intended for Bionic Eye.
}

\author{
Hima Bindu Katikala, Sadulla Shaik, Muralidharan Jayabalan, Yatavakilla Amarendra Nath
}

\begin{abstract}
According to the Survey of WHO [World Health Organization], a people of about 285 million are visually impaired, 39 million are blind and 246 million are having low vision. Blindness is a serious ailment that makes people to suffer a lot. It occurs because of the eye related disorders like Retinitis Pigmentosa[RP], Age-Related Macular Degeneration[AMRD] and Glaucoma, resulting to lead an uncomfortable life style. Recent developments of the Biomedical Engineering field lead to the development of artificial human eye-Bionic eye analogous to natural eye. Bionic eye is a complex system which is integrated with many sub systems such as CCD camera, video processing unit, implantable chip, radio transmitter and receiver. The implanted artificial retina helps the patients of RP and AMRD regains the vision. Apart from this there exist limitations because of electrode count and noise levels. For better optimization to reduce noise levels of bio-potential signals an instrumentation amplifier is used. In this paper different Instrumentation amplifier are discussed.
\end{abstract}

Keywords: Differential Amplifier, CMRR(Common Mode Rejection Ratio), OP-AMP(Operational Amplifier).

\section{INTRODUCTION}

To evaluate the neuron activity it is necessary to measure the weak bio-potential signal and it has to be strengthen using an Amplifier. One of its approach is conceded out by means of the Instrumentation Amplifier[1]. In the basic electronics for amplifying the signals, different devices are used upon which the Differential Amplifier is one shown reducing noise levels at transmission lines. For better amplification the input impedance should be infinite, it is a major limitation in differential amplifier.

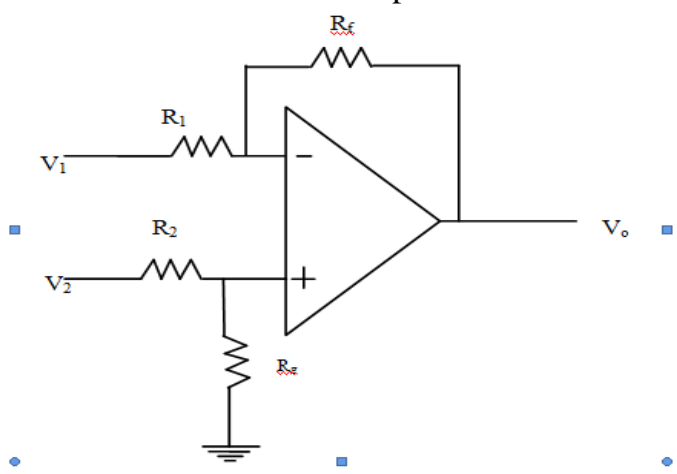

Fig1. Differential Amplifier.

Revised Manuscript Received on December 15, 2019

Hima Bindu Katikala, Ph.D Research Scholar Electronics and Communication Engineering VFSTR (Deemed to be University) Vadlamudi, India. katikala.himabindu@gmail.com

Dr.Sadulla Shaik, Professor \& HoD, Electronics and Communication Engineering KKR and KSR Institute of Technology and Sciences (KITS),Vinjanampadu, India. sadulla09@gmail.com

Dr. Muralidharan Jayabalan, Assoc. Prof. Electronics and Communication Engineering VFSTR (Deemed to be University) Vadlamudi, India. muralidharanae@gmail.com

Yatavakilla Amarendra Nath, Scientist, Assistant Prof. Electronics and Communication Engineering VFSTR (Deemed to be University) Vadlamudi, India. amar.drdo@gmail.com above in Fig1.It amplify the signals with good CMRR by

For accomplish the limitation, the feedback resistance $\left(\mathrm{R}_{\mathrm{f}}\right)$ value taken as large to enhance the gain. By increasing the resistances value it causes

1.Thermal noise - Shrink accuracy.

2.Stray capacitance - Negatively affect CMRR for high frequencies. Because of the above constraints Differential Amplifier is not used for amplifying weak signals. Detecting the Bio-signal with weak amplitude of mv (milli volts), operating at low frequency range below $1 \mathrm{KHz}$ is challenging task, for this purpose the Instrumentation Amplifiers(hereby representing as IA) with the different topologies are used. IA are used to reject the unwanted noise and also strengthen the floating signals. In biomedical field for the measurement of ECG, EEG, EMG, etc., the body electrodes are interfaced with monitoring devices[2].

At this stage the interference of noise signals are added due to environmental conditions, dc offset voltages and device internal noise. Therefore the usage of IA is extremely concerned in biomedical applications for amplification purpose.

The operation of the IA is diverse from Opamp illustrate in Fig2. Foremost divergence is Opamp employee the additional number of external components like R,L,C whereas the IA uses the additional resistor $\left(R_{g}\right)$ for gain purposes with additive resistor network. $\mathrm{V}_{\text {ref }}$ is always grounded for numerous application. The output voltage $V_{\text {out }}$ constantly swing between mid-supply of input voltage applied. IA are used to amplify the microvolt signals by rejecting Common Mode(CM) signals at its input.

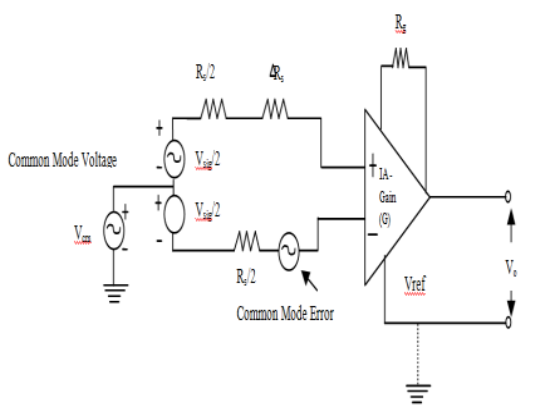

Fig2. General Instrumentation Amplifier.

\section{DIFFERENT BIO-INSTRUMENTATION AMPLIFIERS}

\section{A. IA with Current Feedback Amplifier(CFA) Technique}

For amplification of measured signal essentially in acquisition system it is desired to use the IA with CFA technique [3].The CFA technique consists:-Stage I. The Input with Error amplifier, Stage II.I-V converter and Stage III.The Buffer. 


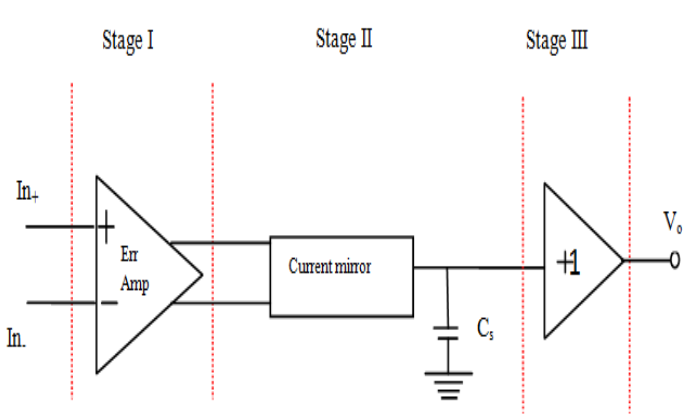

Fig3. Block diagram of CFA.

From Fig3.,Stage I contain-A resistive network used for setting the operating point of two BJT transistors to encompass minimum offset voltage by modifying the noninverting input. I-V converter contain the current mirror to produce constant current despite of loading concern with proper matching of magnitudes and the Stability capacitor $\left(C_{\mathrm{s}}\right)$ makes the circuit stable for different operating points in Stage II. Output Buffer Stage III endow with the Gain current having voltage gain of 1.By using CFA the IA have power dissipation is of $500 \mu \mathrm{V}$, variable Gain and CMRR of $100 \mathrm{~dB}$.

\section{B.IA with Current Balancing Technique(CBT).}

The IA is used in the measurement of ECG. For the measurement of weak signals there exist two aircrafts 1.Baseline wander drift and 2.High frequency noise .The former subsist as of the signal fluctuation arise in the movement of patient body as well as the instrument.

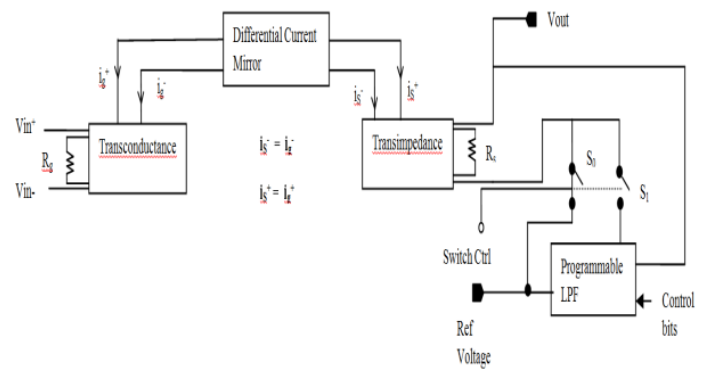

Fig4. Block diagram of CBT.

The immediate remedy carried out by using the IA With Current Balancing Technique [4]. In CBT the programmable filter minimizes the Baseline wander effect as in Fig4. The latter are exterminate by having proper transconductance and impedance matching. By having the above mentioned technique the CMRR is achieved is $100 \mathrm{~dB}$ with Gain of 55.6dB. The amplifier considered with little power consumption $9 \mu \mathrm{W}$ and having a assistance of low cost.

\section{C.IA with Level Shifter Technique( LST)}

For the Biomedical Monitoring Devices the interferences of signal occurs frequently, hence they creates noise levels at the output .Those noises are called as the flicker noise. In the direction of decline those noise level with better CMRR. IA was designed using 3 Opamps, resistors and four current mirror as shown below in Fig5. The proposed circuit have used the PSRR (Power supply rejection ratio) to condense the DC Offset level (noise).

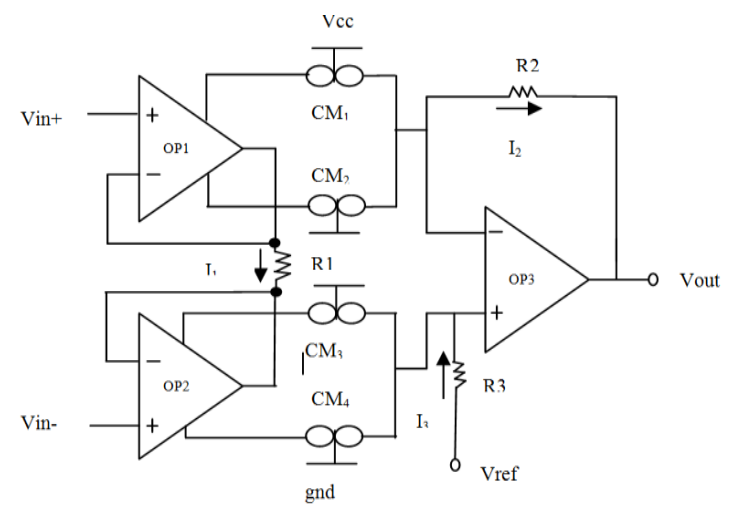

Fig5.IA with OPAMPS.

The Level shifter is used at the input stage to reduce the dc offset .It provides the zero dc offset with amplitude of $5 \mathrm{mv}$ at the output. The capacitive level shifter is simple in design and have less delay, however its limitation is to design properly . In this methodology the level shifter was designed using differential input and differential output which generate the signal using PMOS transistor [5] in its critical path to reduce the flicker noise.

\section{D.IA with Biasing Generator Technique (BGT)}

The probability of dc offset is due to the skin-electrode interface especially for wireless application[9],[10]. To limit the scenario Biomedical signals are to be amplified properly. Zhang(2009) proposed a Instrumentation Amplifier with two major blocks.

1.Current Reference with Bias Generator.

2.Low Offset Op-Amp.

Below Fig 6 illustrate the proposed block.

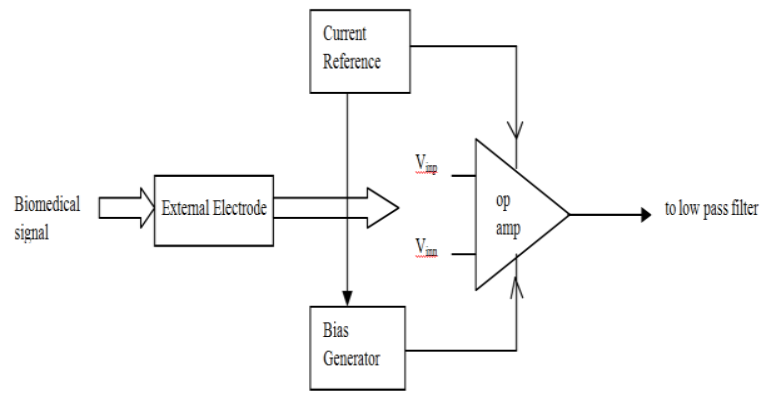

Fig6. Biasing Generator Technique.

The microelectrodes are used for recording the potential which are small in size and having high input impedance especially in visual restoration purpose[6]. Role of biasing is to generate proper DC signal to the Op-Amp which is having two positive and negative signals. The output signal is having high frequency components and eliminated by using low pass filter. However the propagated signal is still having the dc offset which is reduced by the DC Offset Rejection Technique[7]. The power dissipation is of $66 \mu \mathrm{W}$ with CMRR of $100 \mathrm{~dB}$.

\section{RESULT DESCRIPTION}

As of the above mentioned techniques the another processing method used to amplify the Bio-potential signals is by using PGA. It contains Front end amplifier with the input resistance(Ri1 and Ri2)as illustrate in Fig7. 
The former one have the digital control switch, Instrumentation Amplifier and Programmable gain Amplifier.

The signal with strong rejection of interference is the outcome of the IA. For perfect isolation of the flicker noise, a chopper circuit is used and discussed in [8]. Mainly the PGC offers a variable gain along with bandwidth which is controlled with a digital switch. Corresponding Gain is $125 \mathrm{~dB}$ with variable bandwidth, operates at $0.4-0.8 \mathrm{v}$ and having power dissipation of $0.09 \mathrm{~mW}$.

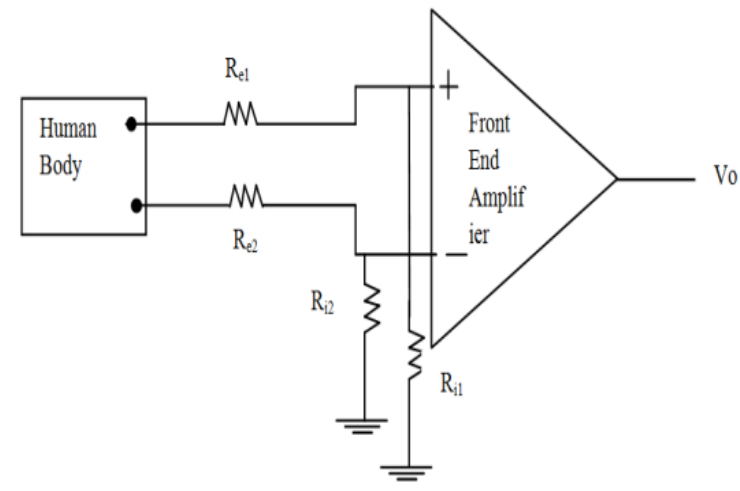

Fig7.IA with PGC.

Table I. Comparison of above mentioned IA.

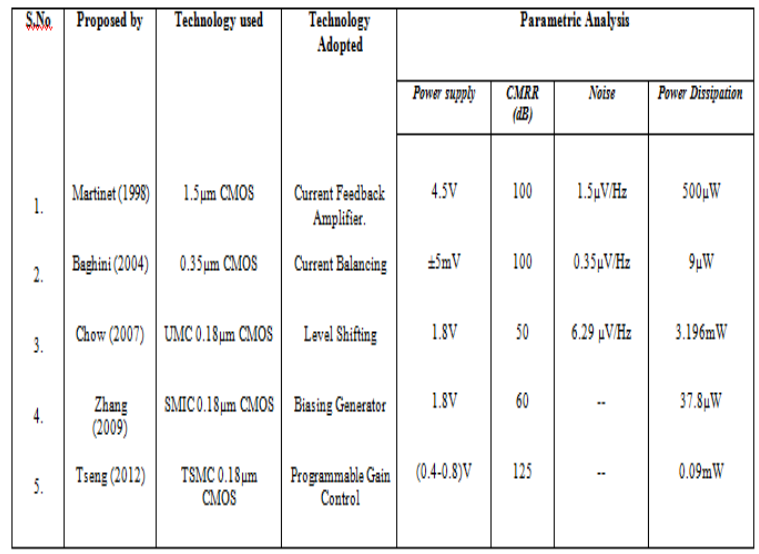

\section{CONCLUSION}

Different approaching methods are to be develop, to replace the damaged photoreceptor by propagate the light to neuron of retina and providing vision through the electrodes. Accompanied by Different technology with various parameters enhances the amplifier signal. In Biomedical field, amplifying the bio-potential stimuli is always challenging. By combining the Instrumentation amplifier technology with bio-potential stimuli, one can achieve the desired output. This paper represents the bio-potential amplified signal with high CMRR and reduce noise level can be achieved by Programmable Gain Control technology.

\section{REFERENCES}

1. Jasbir Kaur , Anisha Ganpati,"A Review on Instrumentation Amplifier and Methods to Improve CMRR", International Journal of Electrical Electronics \& Computer Science Engineering Vol 5, Iss No 1 ,February, 2018

2. G.Sathiyabama,G.Vinudevi,Abhilashini.R, P.Indhupriya," A Survey on Instrumentation Amplifiers used for Biomedical Application", International Journal of Advanced Research in Electrical, Electronics and Instrumentation Engineering Vol. 4, Iss No 3, March 2015.

3. Rui Martins, Siegfried Selberherr, and Francisco A.Vaz, , IEEE,"A CMOS IC for Portable EEG Acquisition Systems", IEEE transactions on instrumentation and measurement, Vol. 47, Iss No. 5 , Pp 1191-1195,October 1998.

4. Shojaei-Baghini M, Lal RK, Sharma DK, "An ultra low-power CMOS instrumentation amplifier for biomedical applications ", IEEE International Workshop on Biomedical Circuits and Systems, December 2004.

5. Chow HC, Wang JY, "High CMRR instrumentation amplifier for biomedical applications",9th International Symposium on Signa Processing and Its Applications, Pp:1-4, February 2007.

6. Hima Bindu Katikala, Dr. Sadulla Shaik "A Survey of the Vision Restoration Eye", Journal of International Pharmaceutical Research,Vol45,IssNo.1 Pg : 501-505,December 2018.

7. Zhang JY, Wang L, Li B, "Design of low-offset low-power CMOS amplifier for biosensor application", Journal of Biomedical Science and Engineering, Vol 2, Iss No 7, Pp: 538-542, November 2009.

8. Tseng,Yuhwai,yingchieh,shuoting kao and Chauchin Su, "A $0.09 \mathrm{uW}$ Low Power Front-End Biopotential Amplifier for Biosignal Recording", IEEE Transaction on Biomedical Circuits and System, Vol 6,Iss No 5,Pp:508-516,October 2012.

9. Sadulla Shaik, M. Pavan kumar, Prathiba Jonnala, G. B. V. Ramulu and Dr. K. Sri Rama Krishna. "Design of Cost effective Wireless Patient Monitoring System Using PIC16F877A." International Journal of Applied Engineering Research, Vol. 10, Iss No. 1, Pp. 380-383,2015.

10. A.V.NageswaraRao, G.S.R.Satyanarayna and Shaik Sadulla, "PSoC Based Wireless Biomedical Monitoring System Using RF" National Conference on VLSI, Signal Processing \& Communications (NCVSComs-12), 2012 